Kevin M. Kelly

Judy Dean

W. Scott Comulada

Sung-Jae Lee

\section{Breast cancer detection using automated whole breast ultrasound and mammography in radiographically dense breasts}

Received: 14 May 2009

Revised: 27 July 2009

Accepted: 7 August 2009

Published online: 2 September 2009

(C) The Author(s) 2009

This article is published with open access at

Springerlink.com

\section{K. M. Kelly $(\bowtie)$}

Huntington Memorial Hospital,

Huntington-Hill Breast Center,

Pasadena, CA, USA

e-mail: drkelly@ca.rr.com

Tel.: +1-626-7936141

Fax: +1-775-2561845

\section{J. Dean}

1525 State St. Suite 102,

Santa Barbara, CA, 93101, USA

e-mail: judy@judydeanmd.net

W. S. Comulada $\cdot$ S.-J. Lee

Semel Institute Center for Community

Health, University of California,

Los Angeles, CA, USA

e-mail: scomulad@ucla.edu

e-mail: sjlee@mednet.ucla.edu
Abstract Purpose: Mammography, the standard method of breast cancer screening, misses many cancers, especially in dense-breasted women. We compared the performance and diagnostic yield of mammography alone versus an automated whole breast ultrasound (AWBU) plus mammography in women with dense breasts and/or at elevated risk of breast cancer. Methods: AWBU screening was tested in 4,419 women having routine mammography (Trial Registration: ClinicalTrials.gov Identifier: NCT00649337). Cancers occurring during the study and subsequent 1-year follow-up were evaluated. Sensitivity, specificity and positive predictive value (PPV) of biopsy recommendation for mammography alone, AWBU and mammography with AWBU were calculated. Results: Breast cancer detection doubled from 23 to 46 in
6,425 studies using AWBU with mammography, resulting in an increase in diagnostic yield from 3.6 per 1,000 with mammography alone to 7.2 per 1,000 by adding AWBU. PPV for biopsy based on mammography findings was $39.0 \%$ and for AWBU $38.4 \%$. The number of detected invasive cancers $10 \mathrm{~mm}$ or less in size tripled from 7 to 21 when AWBU findings were added to mammography. Conclusion:

AWBU resulted in significant cancer detection improvement compared with mammography alone. Additional detection and the smaller size of invasive cancers may justify this technology's expense for women with dense breasts and/or at high risk for breast cancer.

Keywords Mammography · Ultrasound - Breast cancer . Screening $\cdot$ Automated

\section{Introduction}

Despite progress in identifying risk factors and genetic markers for breast cancer, approximately $70-80 \%$ of cases occur in women without known major predictors [1-3]. Population-based screening for early detection of breast cancer is therefore the primary strategy for reducing breast cancer mortality.

Mammography as the standard imaging method for breast cancer screening [4] has resulted in reduced breast cancer mortality. However, the number of cancers escaping detection with mammography is substantial, particularly in dense-breasted women, with sensitivity as low as $30-48 \%$
[5]. Computer-aided detection (CAD) improves sensitivity but does not identify all cancers $[6,7]$. The DMIST trial found an improvement from $55 \%$ to $70 \%$ in cancer detection comparing digital with film mammography [8], suggesting that substantial numbers of cancers are missed even with digital technology. Mammographically missed cancer is a particular problem for women with dense breasts $[5,9]$; Boyd et al. showed that the odds for interval cancers was 17.8 -fold higher in extremely dense breasts compared with fatty breasts [9].

The performance of mammography is reduced for cancer detection in dense-breasted women as mammograms are summation images, with all breast tissue overlapping in 
each view. Cancers may not be visualized because of overlying dense breast tissue [10-11]. Mammography can miss far posterior cancers in the retro-mammary space because of inadequate positioning of deep tissue [10]. Magnetic resonance imaging (MRI) has recently been recommended by the American Cancer Society (ACS) to screen women at very high risk of breast cancer [12]. Though highly sensitive, MRI is costly and may carry risks from the required contrast media [13]. MRI for breast cancer screening has also been characterized by lower specificity, as compared to mammography with a higher rate of false positives, leading to further follow-up MRI and/or image-guided biopsy costs [14-17]. For example, a study by Leach et al. reported MRI specificity of $81 \%$, compared to $93 \%$ specificity in mammography [18]. Griebsch et al. [14] reported MRI as having almost four times more recalls than mammography for women with high familial breast cancer risk, and $70 \%$ of the recalls did not involve cancer. Because of lower specificity and higher cost, compared to mammography, MRI may not be optimal for breast cancer screening.

Ultrasound is an attractive supplement to mammography because it is widely available, relatively inexpensive and well-tolerated by patients [19-21]. Ultrasound images the tissue to the chest wall in all but the very largest patients, and is a cross-sectional technique, displaying tissue without overlap. Early reports using high-resolution ultrasound for breast cancer detection have been promising [22-29], but the time and skill necessary to detect small, non-palpable tumors with hand-held imaging has discouraged widespread use [30, 31]. Berg et al. (ACRIN study) [32] noted that lack of uniformity and shortage of qualified personnel limit wide implementation of handheld imaging.

The automated whole-breast ultrasound (AWBU: Sonocine, Inc., 5475 Reno Corporate Dr., Suite 200, Reno, NV 89511) system gathers standardized uniform image sets by lesser trained personnel, allowing shorter, more efficient time use by physicians interpreting the studies. Previous studies have shown that 3D AWBU is feasible [33, 34]. Previous studies have also shown that $3 \mathrm{D}$ AWBU has excellent interobserver variability with $2 \mathrm{D}$ benign and malignant lesions [35]. The aim of this study was to determine the improvement in diagnostic yield in detecting non-palpable breast cancer in asymptomatic women with 2D AWBU added to mammography.

\section{Materials and methods}

\section{Participants}

From January 2003 to July 2007, women were recruited for AWBU studies at presentation for routine mammography at eight facilities in Pasadena, Redding, Santa Barbara, Orange and Solano Beach, CA; Albuquerque, NM;
Atlanta, GA; and Ogden, UT. Study participation was offered to consecutive asymptomatic women who had BIRADS density 3 or 4 , heterogeneously or extremely radiographically dense breasts, family or personal history of breast cancer, and/or implants, and who were at least 35 years old unless they had a family or personal history of breast cancer. Women with greater than $7 \mathrm{~cm}$ compressed breast thickness at mammography were specifically not recruited because of ultrasound's limited effectiveness in identifying small masses at these depths, and the resultant decreased reliability of AWBU for these women. Asymptomatic participants were individually interviewed, given a detailed verbal explanation of the study protocol and consent, and interviewed to confirm they were asymptomatic. The percentage of women volunteering for AWBU varied by site. Approximately $5 \%$ of those eligible volunteered at sites that only informed the women of the study by mail and required them to make AWBU appointments at the same time as their mammograms. Up to $25 \%$ of women volunteered when informed of the study by personnel at the breast center and offered immediate AWBU in conjunction with their mammograms.

The study consent was approved by the Institutional Review Boards at each hospital, or The Western Institutional Review Board. The consent specifically stated that AWBU is an investigational procedure meant to supplement, not replace, mammography.

A total of 6,425 AWBU examinations and mammograms were performed on 4,419 asymptomatic women; 4,991 of the mammograms were routine screening studies, and 1,434 were annual asymptomatic diagnostic, immediate review studies (22\%), including 776 with previous breast cancer, 399 with implants and 159 with non-localized findings such as diffuse nodularity or diffuse breast tenderness. A third $(36 \%$; $n=2297)$ of the mammograms were digital, and two thirds analog. Participants had the option of having AWBU concurrent with mammography (3,951 women) or alternating mammography and AWBU every 6 months (468 women) according to the preference of the individual woman. Some women who had had previous breast cancer or who had a strong family history of breast cancer preferred to be examined by mammography or AWBU at approximately 6-month intervals. Matching studies were included in the analyses if the examinations fell within a reasonable window, defined as 34 days for participants opting for concurrent screenings and 5-8 months for patients alternating techniques. Participants were included in the analysis if they were asymptomatic and had at least one pair of matching examinations within the allowed times. Six paired examinations were incomplete or probably benign (BIRADS 0 or 3), but the patients had not completed followup and were excluded from the analysis. Fifty patients were excluded for the following reasons: 35 had palpable findings, including 5 palpable cancers; 15 elected AWBU after an abnormal mammogram, including 5 cancers, and 
were excluded because the AWBU interpretation was not blinded to mammography findings. Eleven biopsies were excluded for the following reasons: six patients had biopsies over a year after AWBU, including 2 cancers; four had benign biopsies performed for findings seen with MRI only; one participant had therapeutic seroma drainage.

One of ten radiologists, FDA-qualified in mammography and with at least 10 years' experience in breast ultrasound, interpreted each AWBU study. The mammograms were interpreted by a single radiologist in the usual manner for that institution. The radiologists were blinded to the results of the corresponding mammograms or AWBU studies, and in some cases the same radiologist interpreted both studies.

The mammograms of all participants with cancers detected by AWBU were retrospectively reviewed, ensuring that the original reader had not missed a mammographically detectable cancer. Mammograms and AWBU studies of all participants with clinically detected interval cancers occurring within 1 year of a normal mammogram were similarly reviewed. Mammographic follow-up for at least 1 year since the index imaging was available for 5,089 examinations $(80.0 \%)$.

\section{Diagnostic tool}

AWBU is a computer-based system for performing and recording ultrasound of the whole breast (SonoCine, Reno, NV). The images were collected with multi-frequency transducers within at least the 7 to $12 \mathrm{mHz}$ range. The ultrasound systems used are the Phillips iU22, the Siemens Sequoia, the GE Logiq 9 and the ATL HDI 5000. The transducer is attached to a computer-guided mechanical arm, and images acquired in longitudinal rows (acquiring transverse images), overlapping 7 to $10 \mathrm{~mm}$ to ensure complete coverage. As the transducer faces in more than $95 \%$ of the examinations measured $5.2 \mathrm{~cm}$, the width of the rows without the overlap was about 4.2 to $4.5 \mathrm{~cm}$. In most women the number of rows varied from 4 to 7 for each breast. The mechanical arm controls transducer speed and position, with a trained ultrasound technologist maintaining appropriate contact pressure and orientation vertical to the skin. Approximately 150-300 images per row are immediately displayed on the AWBU monitor and then permanently stored. The interval between recorded images is $0.8 \mathrm{~mm}$. Typical imaging time is $10-20 \mathrm{~min}$ for each participant, with additional participant preparation times of 5-10 min. Interpretation and reporting time for an experienced radiologist was $7-10 \mathrm{~min}$ per examination for typical AWBU studies.

AWBU software creates a cine loop of the images for interpretation, simulating the appearance of real-time imaging. Each cine loop varies from 2,000 to 5,000 images depending on breast size, with about 3,000 images for the average woman. Lesion detection is enhanced by review of the cine loop at about 10 images per second to simulate motion through the breast. Single still images are reviewed only after a possible abnormality has been identified. Any point on an image can be identified as a distance from the nipple in a specific radius using spatial registration recorded as images are acquired. Image review is optimized by playback on a high-resolution monitor allowing compressed image size, three-dimensional reconstruction, and adjustment of contrast, brightness and review speed.

Studies were reported according to the American College of Radiology Breast Imaging Reporting and Data System (BI-RADS) six-point scale $(0=$ incomplete, needs additional assessment; $1=$ normal; $2=$ benign; $3=$ probably benign; $4=$ suspicious; $5=$ highly suggestive of malignancy) $[36,37]$.

Follow-up

Data were collected for size, stage and grade of cancers detected by imaging and during a 1-year follow-up period. When both studies were normal, no further action was required. If the results of AWBU, mammography or both required further action, additional evaluation was completed unless the initial abnormal finding was fully explained by the other imaging study. For examinations scored as 0 or 3 , additional imaging was performed to determine the final BI-RADS assessment.

If the BI-RADS score was 4 or 5 , a stereotactic radiographic or ultrasound directed 14 gauge or larger percutaneous biopsy was performed. If a benign non-highrisk lesion was diagnosed, no further tissue sampling was performed. If a focal high-risk lesion, such as atypical ductal hyperplasia, papillary neoplasm or radial scar, was discovered, the diagnosis was confirmed by surgical removal of the lesion. If a malignancy was found, the lesion was removed surgically by lumpectomy or mastectomy, and a final pathological stage was assigned by the pathologists in the usual manner for that hospital in accordance with the American Joint Committee on Cancer (AJCC) TNM system guidelines. The pathologists were blinded to the patients' participation in the study and the method of cancer detection.

\section{Statistical analysis}

Improvements in diagnostic yield were assessed by calculation of the sensitivity, specificity and positive predictive value (PPV) for biopsy recommendation based on mammography plus AWBU, compared with mammography. Analyses were carried out using SAS version 9.1 software [38]. Exact 95\% confidence intervals (CI) were calculated for diagnostic yield [39]. Chi-square test 
statistic was used to compare the number of cancers detected by AWBU, based on the size of cancer. McNemar's test was conducted to compare diagnostic yield between mammography and AWBU. We compared the PPV between mammography and AWBU following methodology proposed by Leisenring [40]. P-values of less than 0.05 were considered to indicate statistical significance.

\section{Results}

\section{Participants}

Ethnic groups in the study $(\mathrm{n}=4419)$ were whites $(87 \% ; \mathrm{n}=$ $3843)$, Hispanics $(6 \% ; n=248)$, Asians $(3 \% ; n=128)$ and blacks $(1 \% ; n=61)$. More than half of the participants reported never taking hormone replacement therapy $(59 \%$; $\mathrm{n}=2,618) ; 11 \%(\mathrm{n}=500)$ of all participants and 5\% (3 out of 57) of participants found to have breast cancer had implants. Personal history of breast cancer was reported in $10 \%$ of participants $(n=432)$ and first or second degree relatives with breast cancer in $30 \%(n=1,314)$ and $29 \%$ $(n=1,280)$, respectively. Four participants $(0.1 \%)$ have a known mutation of BRCA1/2. None of the abovementioned breast cancer risk factors were reported by $1,279(29 \%)$. Median age of the study population was 53 years $($ range $=24$ to 89 ).

\section{Breast cancer detection}

There were 57 cancers in 56 participants $(1.3 \%$ of the sample); one participant had bilateral cancer. Mean age of participants with breast cancer $(n=56)$ at the time of biopsy was 57 years; 14 cancers occurred in women younger than 50 , and 8 patients were at least 70 . Cancer detection was added for patients in all age groups, but age subsets were too small for statistical analysis. Three quarters (43 out of 57 ) of breast cancers were reported in participants with non-

Table 1 Non-palpable breast cancer detection by imaging method

\begin{tabular}{|c|c|c|c|c|c|c|}
\hline \multirow[t]{2}{*}{ Cancer types } & \multirow{2}{*}{$\begin{array}{l}\text { Number } \\
\text { of cancers } \\
\mathrm{N}\end{array}$} & \multicolumn{2}{|c|}{ Imaged only by AWBU } & \multirow{2}{*}{$\begin{array}{l}\text { Imaged by both AWBU } \\
\text { and mammography } \\
\mathrm{n}(\%)\end{array}$} & \multirow{2}{*}{$\begin{array}{l}\text { Imaged by } \\
\text { mammography only } \\
\mathrm{n}(\%)\end{array}$} & \multirow{2}{*}{$\begin{array}{l}\text { Neither }{ }^{a} \\
\mathrm{n}(\%)\end{array}$} \\
\hline & & $\mathrm{n}(\%)$ & $95 \% \mathrm{CI}^{\mathrm{b}}$ & & & \\
\hline All cancers & 57 & $23(40)$ & $(27.5,54)$ & $15(26)$ & $8(14)$ & $11(19)$ \\
\hline \multicolumn{7}{|l|}{ Stage } \\
\hline 0 & 7 & $1(14)$ & $(0,58)$ & 2 & 4 & 0 \\
\hline 1 & 33 & $17(51.5)$ & $(33.5,69)$ & 10 & 3 & 3 \\
\hline $2 \mathrm{a}$ & 10 & $4(40)$ & $(12,74)$ & 2 & 0 & 4 \\
\hline $2 b$ & 3 & $0(0)$ & $(0,71)$ & 1 & 1 & 1 \\
\hline $3 a$ & 2 & $1(50)$ & $(1,99)$ & 0 & 1 & 1 \\
\hline $3 b$ & 2 & $0(0)$ & $(0,84)$ & 0 & 0 & 2 \\
\hline Ductal carcinoma in situ & 7 & $1(14)$ & $(0,58)$ & $2(28.5)$ & $4(57)$ & $0(0)$ \\
\hline Invasive cancer & 50 & $22(44)$ & $(30,59)$ & $13(26)$ & $4(8)$ & $11(22)$ \\
\hline Invasive ductal & 42 & $18(43)$ & $(28,59)$ & $12(28.5)$ & $3(7)$ & $9(21)$ \\
\hline Invasive lobular & 8 & $4(50)$ & $(16,84)$ & $1(12.5)$ & $1(12.5)$ & $2(25)$ \\
\hline \multicolumn{7}{|l|}{ Grade of invasive cancers ${ }^{c}$} \\
\hline I & 15 & $7(47)$ & $(21,73)$ & $5(33)$ & $1(7)$ & $2(13)$ \\
\hline II & 21 & $13(62)$ & $(38,82)$ & $5(24)$ & $0(0)$ & $3(14)$ \\
\hline III & 13 & $2(15)$ & $(2,45)$ & $2(15)$ & $3(23)$ & $6(46)$ \\
\hline \multicolumn{7}{|l|}{ Size of invasive cancers } \\
\hline $5 \mathrm{~mm}$ or less ${ }^{\mathrm{c}}$ & 3 & $1(33)$ & $(1,90.5)$ & $1(33)$ & $1(33)$ & $0(0)$ \\
\hline 6 to $10 \mathrm{~mm}$ & 18 & $13(72)$ & $(46.5,90)$ & $3(17)$ & $2(11)$ & $0(0)$ \\
\hline 11 to $20 \mathrm{~mm}$ & 20 & $6(30)$ & $(12,54)$ & $8(40)$ & $0(0)$ & $6(30)$ \\
\hline 21 to $50 \mathrm{~mm}$ & 6 & $1(17)$ & $(0,64)$ & $1(17)$ & $1(17)$ & $3(50)$ \\
\hline over $5 \mathrm{~cm}$ & 3 & $1(33)$ & $(1,90.5)$ & $0(0)$ & $0(0)$ & $2(67)$ \\
\hline
\end{tabular}

Abbreviations: AWBU, automated whole breast ultrasound; CI, confidence intervals

${ }^{a}$ Interval cancers detected within a year of negative imaging

$\mathrm{b}_{95} \%$ confidence intervals for additional cancers detected only by AWBU

${ }^{c}$ One tumor was detected as a lymph node metastasis, with no primary tumor identified by mammography, ultrasound or MRI 
familial or familial risk factors; 11 cancers were reported in participants with a personal history and first- or second-degree relatives with breast cancer, 8 cancers were reported in participants with only a personal history, and 24 were reported in participants only reporting relatives with breast cancer. No participants with cancer had a known BRCA mutation. Only three cancers were observed in participants alternating AWBU/mammography screenings every 6 months.

Breast cancer detections doubled from 23 to 46 in 6,425 studies using AWBU with mammography, resulting in an increase in diagnostic yield from 3.6 per 1,000 with mammography alone to 7.2 per 1,000 by adding AWBU (an additional 3.6 per 1,$000 ; 95 \% \mathrm{CI}=2.3-5.4$ ). Sensitivity for mammography alone was $40 \%(95 \% \mathrm{CI}=27.5-54 \% ; 23$ out of 57 cancers), but increased to $81 \%(95 \% \mathrm{CI}=68$ $90 \%$; 46 out of 57 cancers) with the addition of AWBU. Sensitivity of AWBU alone was $67 \%(95 \% \mathrm{CI}=53-79 \%$; 38 out of 57).

Table 1 shows significant increases in invasive ductal cancer detections; 18 out of 42 invasive ductal cancers were demonstrated by AWBU only (95\% CI $=28-59 \%)$, among grade I invasive cancers $(95 \% \mathrm{CI}=21-73 \%)$ and grade II invasive cancers $(95 \% \mathrm{CI}=38-82 \%)$. Additional grade III invasive cancer detections were $15 \%(95 \% \mathrm{CI}=2-45 \%)$. Significantly smaller invasive tumors were detected by AWBU; 14 out of 21 ( $p=0.006)$, or $67 \%$ of cancers less than $10 \mathrm{~mm}$ or less were detected only by AWBU and not evident by mammography. The sensitivity of AWBU for these tumors was $81 \%$ compared with $33 \%$ for mammography. Cancer detections for invasive tumors measuring 11 to $20 \mathrm{~mm}$ increased from 8 to 14 with the addition of AWBU. Selected AWBU and mammography images of a woman with dense breasts are shown in Fig. 1, demonstrating bilateral cancers evident with AWBU but not mammography.

For women with dense or extremely dense breasts (Table 2), AWBU detected 32 out of 49 cancers or $65 \%$, compared with 19 out of 49 , or $39 \%$ for mammography alone $(p=0.02)$. Addition of AWBU to mammography more than doubled cancer detections from 19 to 39 .

AWBU resulted in more recalls for additional imaging (Table 3$)$ than mammography $(p<0.001)$. Recalls were $4.2 \%$ for screening mammography and $7.2 \%$ for AWBU. Recalls increased from 4.2 to $9.6 \%$ adding AWBU to mammography, as some AWBU-recalled participants were the same participants recalled because of mammography findings. The 208 screening mammography recalls resulted in 13 cancer diagnoses (yield 6.25\%); 465 AWBU recalls resulted in 38 cancer diagnoses (yield 8.2\%).

Specificity based on recalls was $89.9 \%(95 \% \mathrm{CI}=89.1-$ 90.6\%) for $\mathrm{AWBU}, 95.15 \%(95 \% \mathrm{CI}=94.6-95.7 \%)$ for mammography and $98.7 \%(95 \% \mathrm{CI}=98.35-98.9 \%)$ for combined mammography/AWBU. Table 4 outlines the likelihood of cancer per biopsy recommendation, i.e., PPV of cancer. No difference in the probability of cancer was found according to the type of diagnostic test leading to biopsy.
Fig. 1 Selected images of a 54year-old asymptomatic woman with dense breasts and no previous history of breast cancer. a Craniocaudal digital mammograms taken the same day as the AWBU study. b Mediolateral oblique digital mammograms taken the same day as the AWBU study. c Transverse AWBU image of the right breast at $11: 30,6 \mathrm{~cm}$ from the nipple; white arrow shows a $7 \mathrm{~mm}$, grade I, stage 1, invasive ductal carcinoma. d AWBU image of the left breast at 12:00, $3 \mathrm{~cm}$ from the nipple; two white arrows show $10 \mathrm{~mm}$, grade I, stage 1 , invasive carcinoma with lobular carcinoma in situ
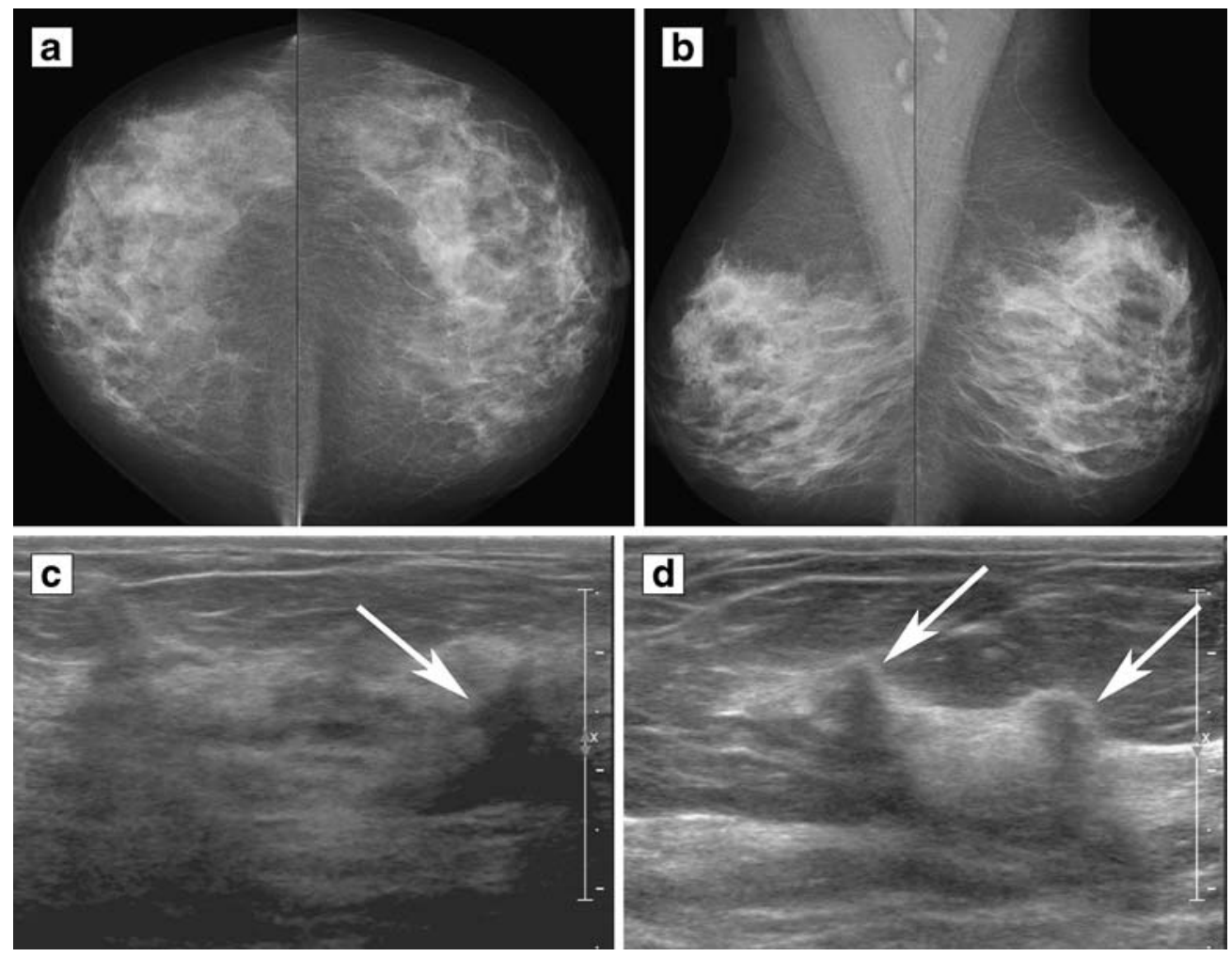
Table 2 Non-palpable breast cancer detections by imaging methods and breast density

\begin{tabular}{|c|c|c|c|c|c|c|c|c|}
\hline \multirow{2}{*}{$\begin{array}{l}\text { Breast density } \\
\text { according to } \\
\text { BI-RADS }\end{array}$} & \multirow{2}{*}{$\begin{array}{l}\text { Number } \\
\text { of patients } \\
\mathrm{N}(\%)\end{array}$} & \multirow{2}{*}{$\begin{array}{l}\text { Number of } \\
\text { cancers } \\
\mathrm{N}\end{array}$} & \multicolumn{2}{|c|}{$\begin{array}{l}\text { Imaged only } \\
\text { by AWBU }\end{array}$} & \multirow{2}{*}{$\begin{array}{l}\text { Imaged by both } \\
\text { AWBU and mammography } \\
\mathrm{n}(\%)\end{array}$} & \multirow{2}{*}{$\begin{array}{l}\text { Imaged by } \\
\text { mammography only } \\
\text { n (\%) }\end{array}$} & \multirow{2}{*}{$\begin{array}{l}\text { Neither }{ }^{a} \\
\mathrm{n}(\%)\end{array}$} & \multirow[t]{2}{*}{$p$ value $^{\mathrm{b}}$} \\
\hline & & & $\mathrm{n}(\%)$ & $95 \% \mathrm{CI}^{\mathrm{c}}$ & & & & \\
\hline 1 (fatty) & $148(2)$ & 2 & $1(50)$ & $(1,99)$ & $1(50)$ & $0(0)$ & $0(0)$ & $\mathrm{d}$ \\
\hline 2 (mixed) & $1,930(30)$ & 6 & $2(33)$ & $(4,78)$ & $2(33)$ & $1(17)$ & $1(17)$ & 1.00 \\
\hline 3 (dense) & $3,923(61)$ & 42 & $15(36)$ & $(21.5,52)$ & $11(26)$ & 7 (17) & $9(21)$ & 0.13 \\
\hline 4 (ex dense) & $424(6.5)$ & 7 & $5(71)$ & $(29,96)$ & $1(14)$ & $0(0)$ & $1(14)$ & 0.06 \\
\hline $3 \& 4$ & $4,347(68)$ & 49 & $20(41)$ & $(27,56)$ & $12(24)$ & $7(14)$ & $10(20)$ & 0.02 \\
\hline
\end{tabular}

Abbreviations: AWBU, automated whole breast ultrasound; ex dense, extremely dense

${ }^{a}$ Interval cancers diagnosed within 1 year of negative mammography and AWBU

${ }^{\mathrm{b}} \mathrm{p}$ value for comparison of number of cancers detected by AWBU versus mammography

$95 \%$ confidence intervals for additional cancers detected only by AWBU

d p value not calculated because of zero cell counts

\section{Retrospective review}

The only detected invasive cancer over $5 \mathrm{~cm}$ was a nonpalpable invasive lobular carcinoma, evident by AWBU but not visible even in retrospect with mammography. Another large invasive lobular cancer that occurred early in the study was not detected prospectively by either mammography or AWBU, but in retrospect is evident by AWBU.

Review of the interval cancers detected clinically within a year of negative imaging showed 9 out of 11 were evident in retrospect by AWBU, and 1 of these was also evident in retrospect by mammography. Two interval cancers were not evident on either mammography or AWBU.

\section{Discussion}

AWBU with mammography is significantly better than mammography alone for detecting breast cancer, espe- cially for dense-breasted women. These women face a particularly difficult situation, as they may be at higher risk of developing breast cancer [41] and less likely to have the cancer detected by standard mammography screening [9-11].

The improvement in cancer detection of 3.6 per 1,000 demonstrated with AWBU is similar to supplemental yield findings of earlier studies using hand-held ultrasound (range $=2.7$ to 4.6 per 1,000 ) [42]. However, AWBU has several advantages over handheld ultrasound. It (1) is more reproducible, thorough imaging through the entire breasts; (2) has higher definition with better contrast and sharpness and smaller images for review by using a high resolution 2,000 line reading monitor with 3D capability and (3) allows delayed interpretation at computer monitor-based read stations with non real-time review, optimizing the radiologist's reading environment.

As $82 \%$ of interval cancers detected clinically before the next screening are apparent retrospectively on

Table 3 Patient recalls for additional studies by initial imaging method

\begin{tabular}{|c|c|c|c|}
\hline Imaging method & $\begin{array}{l}\text { BI-RADS } 0 \\
\mathrm{n} / \mathrm{N}, \%\end{array}$ & $\begin{array}{l}\text { BI-RADS } 3^{a} \\
n / N, \%\end{array}$ & $\begin{array}{l}\text { BI-RADS } 4 \text { or } 5^{a} \\
n / N, \%\end{array}$ \\
\hline All mammograms ${ }^{b}$ & $208 / 6425,3.2 \%$ & $39 / 6425,0.6 \%$ & $59 / 6425,0.9 \%$ \\
\hline Screening mammograms & $208 / 4991,4.2 \%$ & $39 / 4991,0.8 \%$ & \\
\hline AWBU & $465 / 6425,7.2 \%$ & $77 / 6425,1.2 \%$ & $99 / 6425,1.5 \%$ \\
\hline Both mammography and AWBU ${ }^{\mathrm{c}}$ & $50 / 6425,0.8 \%$ & $10 / 6425,0.15 \%$ & $24 / 6425,0.4 \%$ \\
\hline AWBU only ${ }^{d}$ & $415 / 6425,6.45 \%$ & $67 / 6425,1.0 \%$ & $75 / 6425,1.2 \%$ \\
\hline
\end{tabular}

Abbreviations: AWBU, automated whole breast ultrasound

${ }^{a}$ Class 0 imaging studies changed to class 3,4 , or 5 after evaluation

b Includes screening and diagnostic mammograms

${ }^{\mathrm{c}}$ Studies where additional imaging was recommended based on both mammography and AWBU findings independently

${ }^{\mathrm{d}}$ Studies with class 1 or 2 mammography, where additional imaging would not otherwise have been recommended 
Table 4 Positive predictive value of biopsy recommendation by imaging method/s

\begin{tabular}{lllll}
\hline Imaging method/s & Number of cancers & Number of biopsies & Percentage positive & $95 \%$ CI \\
\hline All positive mammograms & 23 & 59 & $39.0 \%$ & 26.6 to $52.6 \%$ \\
All positive AWBU & 38 & 99 & $38.4 \%$ & 28.8 to $48.7 \%$ \\
AWBU positive, mammogram negative & 23 & 75 & $30.7 \%$ & 20.5 to $42.4 \%$ \\
Mammography and AWBU positive & 15 & 24 & $62.5 \%$ & 40.6 to $81.2 \%$ \\
AWBU negative, mammogram positive & 8 & 35 & $22.9 \%$ & 10.4 to $40.1 \%$ \\
\hline
\end{tabular}

Abbreviations: AWBU, automated whole breast ultrasound; CI, confidence intervals

previous AWBU examinations, we postulate that cancer detection with AWBU may improve with increased reader experience and possibly a CAD system. In addition, available previous comparison studies may contribute to better sensitivity and fewer recalls. Assuming that many of the AWBU "missed" cancers could potentially be avoided, AWBU with mammography could have sensitivity approaching $95 \%$. This may be comparable to the sensitivity of MRI, but at a fraction of the cost [14, 15, 43, 44].

The $38 \%$ PPV of biopsy for AWBU observed in this study is substantially higher than the $11 \%$ PPV of biopsy prompted by ultrasound in the ACRIN Trial [31], and higher than the 33\% PPV from the Breast Cancer Surveillance Consortium (BCSC) report [45]. Perhaps our improvement in PPV occurred because the women with suspicious findings on either mammography or ultrasound screening were treated as any other recall patient in the usual manner for the particular imaging department. Consequently, the PPV found in this study is consistent with the PPV found in our institutions in general. Similarly, the recalled women who were not biopsied were assigned to a BI-RADS category of 1 (normal), 2 (benign) or 3 (probably benign). The category-3 women underwent appropriate follow-up, and the others returned to routine screening.

Additionally, only $71 \%$ of invasive cancers detected by the BCSC were smaller than $20 \mathrm{~mm}$, while in this study $90 \%$ were smaller than $20 \mathrm{~mm}$ [45]. The smaller size of invasive cancers detected by adding AWBU to mammography suggests that this technology may have the potential to impact breast cancer survival and treatment options, though this has not yet been shown.

AWBU was well tolerated by participants and easily incorporated into breast imaging practice. Many women who are at increased risk of breast cancer do not meet the ACS criteria for annual MRI, but could benefit from more effective screening than mammography alone. The ease of use and cost at approximately $\$ 300$ make AWBU an attractive alternative to MRI for women with dense breasts, family or personal history of breast cancer, or other risk factors. AWBU is well accepted by participants because of less breast compression than mammography, no exposure to ionizing radiation and no contrast medium injection.

A limitation of our study is the relatively small number of participating facilities and radiologists. Another possible limitation is that AWBU images were collected in transverse (axial) planes. However, 3D reconstruction in coronal, sagittal or any off-axis planes was possible for any discovered possible abnormality. The technique itself is limited, as are other ultrasound techniques, in women with large breasts. Patient cooperation is also necessary as motion beyond quiet breathing will degrade the examination. Plans to expand this research include more centers and a reader study. Continuing modification of the AWBU system has already produced fewer recalled participants, and a CAD system is being developed.

The number of recalls for additional imaging and/or requiring supplemental hand-held ultrasound is a drawback of this technology. This difference is partially explained by the higher cancer detection rate for AWBU compared with mammography, and partially by the availability of comparison AWBU examinations for only $31 \%$ of studies. On the other hand, nearly all mammograms had previous comparison studies. The similar PPV for biopsy recommendation based on AWBU detections compared with mammography findings indicates that AWBU does not lead to an excess of false-positive biopsies, despite the higher recall rate.

The interval cancer rate of $19.3 \%$ demonstrated in this study is also concerning. In spite of a $100 \%$ increase in cancer detections, the interval cancer rate is similar to the ACRIN trial [32], which showed only a 55\% increase in detections. Most of the cancers missed at screening were high grade at the time of discovery. However, 9 of the 11 cancers initially missed with AWBU are visible retrospectively and are generally similar in appearance to the small cancers that were discovered prospectively.

As the study population in both these trials consisted of difficult-to-image, high-risk subjects, these numbers should not be compared with the general screening population. 
However, we do anticipate low sensitivity for cancer detection with mammography with this type of study population, as the imaging characteristics of cancers in the normal-risk, densebreasted population are most likely the same as in a higher risk similar population.

\section{Conclusion}

Limiting AWBU examinations to a high-risk group with dense breasts, similar to the BRCA1/2 studies with MRI $[40,41]$, would dramatically reduce the cost per cancer diagnosis. In our study $87 \%$ of cancer detections added by AWBU were found in the $68 \%$ of studies in women with dense/very dense breasts. Further study should focus on better defining the combination of risk factors and imaging characteristics that warrant supplementation of mammography with AWBU.
Acknowledgements Additional contributions: We thank Wendie A. Berg, MD, PhD, American Radiology Services, Inc., Johns Hopkins Green Spring, Lutherville, Maryland, for her review of the manuscript and helpful comments. We thank the sites, participants and radiologists for their support and participation in this study. The radiologist-readers, other than the authors, were David C. Bryan, MD, Jon M. Foran, MD, M. Linda Sutherland, MD, Jeannie Stryker, MD, Beth D. Kruse, MD, Gary Wood, MD, Catherine J. Babcook, MD, and Richard S. Steffens, MD.

Financial disclosures: This study was supported by Sonocine, Inc. Dr. Kelly is the majority shareholder of Sonocine, Inc. Dr. Dean owns shares in SonoCine, Inc. Neither author has received any form of payment from the company. Statistical analyses were conducted by independent researchers, Drs. Comulada and Lee, who have no potential conflict of interest with respect to this paper.

Open Access This article is distributed under the terms of the Creative Commons Attribution Noncommercial License which permits any noncommercial use, distribution, and reproduction in any medium, provided the original author(s) and source are credited.

\section{References}

1. Colditz GA, Willett WC, Hunter DJ et al (1993) Family history, age, and risk of breast cancer. Prospective data from the Nurses' Health Study. JAMA 270:338-343

2. Gail MH, Brinton LA, Byar DP et al (1989) Projecting individualized probabilities of developing breast cancer for white females who are being examined annually. J Natl Cancer Inst 81:187986

3. Seidman H, Stellman SD, Mushinski MH (1982) A different perspective on breast cancer risk factors: some implications of the nonattributable risk. CA Cancer J Clin 32:301-313

4. Shapiro S, Venet W, Strax P, Venet L, Roeser R (1982) Ten to 14-year effect of screening on breast cancer mortality. J Natl Cancer Inst 69:349-355

5. Mandelson MT, Oestreicher N, Porter PL et al (2000) Breast density as a predictor of mammographic detection: comparison of interval- and screendetected cancers. J Natl Cancer Inst 92:1081-1087

6. Dean JC, Ilvento CC (2006) Improved cancer detection using computer-aided detection with diagnostic and screening mammography: Prospective study of 104 cancers. Am J Roentgenol 187:2028

7. Ho WT, Lam PWT (2003) Clinical performance of computer-assisted detection (CAD) system in detecting carcinoma in breasts of different densities. Clin Rad 58:133-136
8. Pisano ED, Gastonis C, Hendrick E et al (2005) Diagnostic performance of digital versus film mammography for breast cancer screening. $N$ Engl J Med 353:1-11

9. Boyd NF, Guo H, Martin LJ et al (2007) Mammographic density and the risk and detection of breast cancer. $\mathrm{N}$ Engl J Med 356:227-36

10. Bird RE, Wallace TW, Yankaskas BC (1992) Analysis of cancers missed at screening mammography. Radiology 184:613-617

11. Birdwell RL, Ikeda DM, O'Shaughnessy KF, Sickles EA (2001) Mammographic characteristics of 115 missed cancers later detected with screening mammography and the potential utility of computer-aided detection. Radiology 219:192-202

12. Saslow D, Boetes C, Burke W et al (2007) American Cancer Society guidelines for breast screening with MRI as an adjunct to mammography. CA Cancer J Clin 57:75-89

13. Lin SP, Brown JJ (2007) MR contrast agents: physical and pharmacologic basics. J Magn Reson Imaging. 25:884-899

14. Griebsh I, Brown J, Boggis C et al (2006) Cost-effectiveness of screening with contrast enhanced magnetic resonance imaging vs X-ray mammography of women at a high familial risk of breast cancer. Br J Cancer 95:801-810

15. Kuhl CK, Schrading S, Bieling HB et al (2007) MRI for diagnosis of pure ductal carcinoma in situ: a prospective observational study. Lancet 370:485492
16. Schmutzler RK, Rhiem K, Breuer P et al (2006) Outcome of a structured surveillance programme in women with a familial predisposition of breast cancer. Eur J Cancer Prev 15:483-489

17. Kriege M, Brekelmans CT, Boetes C et al (2004) Efficacy of MRI and mammography for breast cancer screening in women with a familial or genetic predisposition. N Engl J Med 351:427500

18. Leach MO, Boggis CR, Dixon AK et al (2005) Screening with magnetic imaging and mammography of a UK population at high familial risk of breast cancer: A prospective mluticentre cohort study (MARIBS). Lancet 365:1769-1778

19. Liberman L, Feng TL, Dershaw DD, Morris EA, Abramson AF (1998) USguided core breast biopsy: use and costeffectiveness. Radiology 208:717-723

20. Mendelson EB, Tobin CE (1995) Critical pathways in using breast US. Radiographics 15:935-945

21. Parker SH, Jobe WE, Dennis MA et al (1993) US guided automated large-core breast biopsy. Radiology 187:507-511

22. Benson SR, Blue J, Judd K, Harman JE (2004) Ultrasound is now better than mammography for the detection of invasive breast cancer. Am J Surg 188:381-385

23. Berg WA, Gutierrez L, Nessaiver MS et al (2004) Diagnostic accuracy of mammography, clinical examination, US, and MR imaging in preoperative assessment of breast cancer. Radiology 233:830-849 
24. Crystal P, Strano SD, Shcharynski S, Koretz MJ (2003) Using sonography to screen women with mammographically dense breasts. Am J Roentgenol 181:177-182

25. Kaplan SS (2001) Clinical utility of bilateral whole-breast US in the evaluation of women with dense breast tissue. Radiology 221:641-649

26. Kolb TM, Lichy J, Newhouse JH (1998) Occult cancer in women with dense breasts: detection with screening US-diagnostic yield and tumor characteristics. Radiology 207:191-199

27. Kolb TM, Lichy J, Newhouse JH (2002) Comparison of the performance of screening mammography, physical examination, and breast US and evaluation of factors that influence them: an analysis of 27,825 patient evaluations. Radiology 225:165-175

28. Leconte I, Feger C, Galant C et al (2003) Mammography and subsequent whole-breast sonography of nonpalpable breast cancers: the importance of radiologic breast density. Am J Roentgenol 180:1675-1679

29. Moon WK, Noh DY, Im JG (2002)

Multifocal, multicentric, and contralateral breast cancers: bilateral whole-breast US in the preoperative evaluation of patients. Radiology 224:569-576

30. Buchberger W, DeKoekkoik-Doll P, Springer P, Obrist P, Dunser M (1999) Incidental findings on sonography of the breast: clinical significance and diagnostic workup. Am J Roentgenol 173:921-927
31. Kopans DB (1999) Breast cancer screening with ultrasonography. Lancet 354:2096-2097

32. Berg WA, Blume JD, Cormack JB et al (2008) Combined screening with ultrasound and mammography vs mammography alone in women at elevated risk of breast cancer. JAMA 299:21512163

33. Chou YH, Tiu CM, Chiang HR, Chen SP, Chiou HJ, Chiou SY (2006) Ultrasound ACR BI-RADSR Categories Applied in an Automated Breast Ultrasound System: Diagnostic Reliability. Radiological Society of North America scientific assembly and annual meeting program. Oak Brook, Ill: Radiological Society of North America

34. Destounis S, Young W, Hanson S, Somerville P, Murphy P, Zuley M (2005) Automated Breast Ultrasound: A Pilot Study. Radiological Society of North America scientific assembly and annual meeting program. Oak Brook, Ill: Radiological Society of North America

35. Wenkel E, Heckmann M, Heinrich M et al (2008) Automated breast ultrasound: lesion detection and BI-RADS classification-a pilot study. Rofo 9:804-808

36. D'Orsi CJ, Bassett LW, Berg WA et al (2003) Breast Imaging Reporting and Data system, BI-RADS: mammography, 4th edn. American College of Radiology, Reston, VA

37. Mendelson EB, Baum JK, Berg WA, Merritt CRB, Rubin E (2003) Breast Imaging Reporting and Data System, BI-RADS: Ultrasound, 1st edn. American College of Radiology, Reston, VA
38. SAS Institute (2004) SAS/STAT user's guide, version 9.1. SAS Publishing, Cary, NC

39. Mayo Foundation for Medical Education and Research. Locally Written SAS Macros. Mayo Clinic Web Site. Accessed April 4, 2009, at http://mayoresearch. mayo.edu/mayo/research/biostat/ sasmacros.cfm

40. Leisenring W, Alonzo T, Pepe MS (2000) Comparisons of predictive values of binary medical diagnostic tests for paired designs. Biometrics 56:345-351

41. Boyd NF, Byng JW, Jong RA et al (1995) Quantitative Classification of mammographic densities and breast cancer risk: results from the Canadian National Breast Screening Study. J Natl Cancer Inst 87:670-675

42. Berg W (2009) Tailored supplemental screening for breast cancer: What now and what next? Am J Roentgenol 192:390-399

43. Tilanus-Linthorst MMA, Obdeijn IMM, Bartels KCM, deKonig HJ, Oudkerk M (2000) First experiences in screening women at high risk for breast cancer with MR imaging. Breast Cancer Res Treat 63:53-60

44. Plevritis SK, Kurian AW, Sigal BM et al (2006) Cost-effectiveness of screening BRCA1/2 mutation carriers with breast magnetic resonance imaging. JAMA 295:2374-2384

45. Weaver DL, Rosenberg RD, Barlow WE et al (2006) Pathologic Findings from the Breast Cancer Surveillance Consortium: Population-Based outcomes in women undergoing biopsy after screening mammography. Cancer 106:732-42 